\title{
Pupil Ovalization and Accommodation Loss after High-intensity Focused Ultrasound Treatment for Glaucoma: A Case Report
}

\author{
Amado Rivero-Santana ${ }^{1}$, David Pérez-Silguero ${ }^{2}$, Miguel A Pérez-Silguero ${ }^{3}$, Pablo Encinas-Pisa ${ }^{4}$
}

\begin{abstract}
Aim: The aim of this study is to present a complication derived from high-intensity focused ultrasound (HIFU) not described previously in the literature.

Background: HIFU has shown to be an effective technique for reducing intraocular pressure (IOP) in the treatment of glaucoma, enabling the selective coagulation of the ciliary body. Published trials have not observed major intra- or postoperative complications, or serious adverse events derived from this technique.

Case description: We present the case of a 65 -year-old phakic patient with open-angle glaucoma and no previous filtration surgery. He was treated in both eyes with HIFU. After the intervention, the patient presented mild uveitis for 10 days, which remitted with the application of topical cycloplegic and corticosteroid treatment. After that, he reported a loss of near vision and examination showed pupil ovalization. It persisted 6 months later, with an accommodation loss of one diopter.

Conclusion: Phakic patients treated with HIFU might be at risk of pupil ovalization with accommodation loss.

Clinical significance: The complications described in this article should be included in the informed consent for patients with glaucoma who are offered HIFU as a treatment option.

Keywords: Accommodation loss, Glaucoma, High-intensity focused ultrasound, Pupil ovalization.

Journal of Current Glaucoma Practice (2019): 10.5005/jp-journals-10078-1256
\end{abstract}

\section{BACKGROUND}

Circular cycloablation by means of ultrasound is a technique which enables the selective coagulation of the ciliary body. Its efficacy and safety have been published, both in patients with and without previous filtration surgery. ${ }^{1-4}$

We present a complication derived from this technique, to our knowledge not previously described in the literature.

\section{Case Description}

A 65-year-old male phakic, patient came to our service with a previous diagnosis of open-angle glaucoma, and intolerance to all topical antiglaucomatous treatments he had been prescribed. At that moment, he was being treated with topical latanoprost and brinzolamide. IOP was $22 \mathrm{~mm} \mathrm{Hg}$ in the right eye (RE) and $24 \mathrm{~mm} \mathrm{Hg}$ in the left eye (LE), measured with the Goldmann tonometer, and pachymetry yielded values of 530 and 541 microns, respectively. A surface study was carried out with a keratograph (OCULUS Optikgerate GmbH, Wetzlar, Germany) and the following results were obtained: noninvasive keratograph break-up time (Nikbut): 3 seconds (RE); 4 seconds (LE); ocular surface disease index (Osdi test, range 0-100): 38; tear meniscus height: $0.2 \mathrm{~mm}$; osmolarity 315-320 (Tearlab), and examination with slit lamp revealed corneal punctate epitheliopathy in the lower corneal area.

Due to the poor condition of the ocular surface, we ruled out invasive procedures and treatment with HIFU was planned, by means of the Eye-OP1 ${ }^{\circledR}$ device (Eye Tech Care, EyetechcareRillieux-la-Pape, France). After signing the informed consent, the procedure was performed in both eyes, with a $12 \mathrm{~mm}$ probe.
${ }^{1}$ Department of Health Technology Assessment, Fundación Canaria de Investigación Sanitaria (FUNCANIS), Santa Cruz de Tenerife, Spain

${ }^{2,4}$ Department of Ophthalmology, Pérez-Silguero Ophthalmologic Clinic, Las Palmas de Gran Canaria, Spain

${ }^{3}$ Department of Ophthalmology, La Paloma Hospital, Las Palmas de Gran Canaria, Spain

Corresponding Author: Amado Rivero-Santana, Department of Health Technology Assessment, Fundación Canaria de Investigación Sanitaria (FUNCANIS), Santa Cruz de Tenerife, Spain, Phone: +34 922684019 (ext 246), e-mail: amado.riverosantana@sescs.es

How to cite this article: Rivero-Santana A, Pérez-Silguero D, et al. Pupil Ovalization and Accommodation Loss after High-intensity Focused Ultrasound Treatment for Glaucoma: A Case Report. J Curr Glaucoma Pract 2019;13(2):77-78.

Source of support: Nil

Conflict of interest: None

Treatment consisted in the sequential activation of six piezoelectric transducers for 8 seconds.

After the intervention, the patient presented mild anterior uveitis for 10 days, more than usual with this treatment according to our experience, 10 cases of mild uveitis, with a maximal duration of 3 days, among 42 pseudophakic patients treated (non-published data). It remitted with topical cycloplegic and corticosteroid treatment. After withdrawing this and the usual period of clearance (14 days after the HIFU treatment), the patient reported loss of near vision. Biomicroscopy showed pupillary ovalization, which persisted to the same degree 6 months later (Fig. 1), with a 

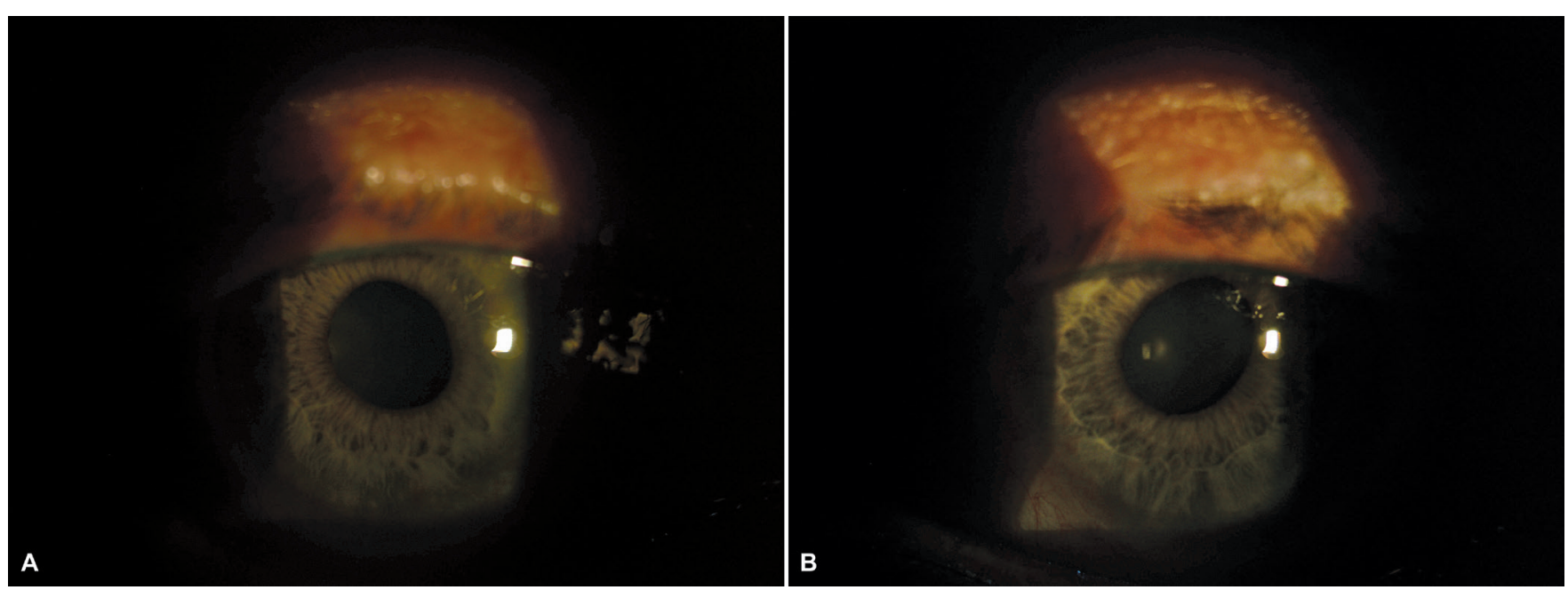

Figs $1 \mathrm{~A}$ and B: Pupil ovalization. (A) Right eye; (B) Left eye

normoreactive pupil. Near visual acuity did not recover the levels previous to the intervention, presenting an accommodation loss of 1 diopter, according to Donders' test. The IOP during the first week decreased to $11.5 \mathrm{~mm} \mathrm{Hg}(\mathrm{RE})$ and $12.5 \mathrm{~mm} \mathrm{Hg}(\mathrm{LE})$, but after disappearing of the uveitis, it increased to 19.5 and $17.5 \mathrm{~mm} \mathrm{Hg}$, respectively. At 6 months, values were 25 and $24 \mathrm{~mm} \mathrm{Hg}$, without topical treatment.

\section{Discussion}

HIFU treatment applied in this study consisted in the sequential activation of 6 miniaturized piezoelectric transducers, activated for 8 seconds. At the histological level, it seems to produce segmental-toannular lesions in the ciliary processes, mainly by coagulation necrosis, respecting the sclera and lens and with limited inflammation. ${ }^{5}$ Findings in rabbits by Aptel et al. ${ }^{6}$ suggest that HIFU cyclocoagulation might have a dual effect on aqueous humor dynamics, inducing degeneration or necrosis and subsequent sloughing off of the ciliary processes' epithelium, on the one hand, and increasing the aqueous outflow by the uveoscleral pathway, on the other hand.

Its clinical efficacy has been confirmed in several publications, both in glaucoma without previous surgery and refractory glaucoma, with mean IOP reductions ranging from 27.8 to $30 \%,{ }^{1-4}$ and even higher when treatment was applied twice $(34.7 \%$ reduction) and 3 times (52\%). ${ }^{3}$

The complications observed in our patient, according to our knowledge, have not been previously described. We believe that the fact of being a phakic patient is the key factor that explains the accommodation loss and pupillary ovalization. The root of the iris, the ciliary body, and the zonula are functionally and anatomically related structures. Pupillary ovalization was oblique in both eyes, with a major axis in the superior temporal-inferior nasal direction. In these two positions, 2 out of the 6 piezoelectric transducers were activated in the treatment. He was the only phakic patient treated with HIFU in our service (among a total of 42 pseudophakic patients). Interestingly, most publications do not distinguish between phakic and pseudophakic patients, and at the light of this finding this distinction should be considered.

\section{ConClusion}

Phakic patients treated with HIFU might be at risk of pupil ovalization with accommodation loss.

\section{Clinical Significance}

The complications described in this article should be included in the informed consent for patients with glaucoma who are offered HIFU as a treatment option.

\section{Patient Consent for Publication}

The patient signed a consent form for publication of the article on 4 October 2018.

\section{References}

1. Denis P, Aptel F, et al. Cyclocoagulation of the ciliary bodies by highintensity focused ultrasound: a 12-month multicenter study. Invest Ophthalmol Vis Sci 2015 Jan 20;56(2):1089-1096. DOI: 10.1167/iovs.1414973.

2. Giannaccare G, Vagge A, et al. High-intensity focused ultrasound treatment in patients with refractory glaucoma. Graefes Arch Clin Exp Ophthalmol 2017 Mar;255(3):599-605. DOI: 10.1007/s00417-0163563-z.

3. De Gregorio A, Pedrotti E, et al. Safety and efficacy of multiple cyclocoagulation of ciliary bodies by high-intensity focused ultrasound in patients with glaucoma. Graefes Arch Clin Exp Ophthalmol 2017 Dec;255(12):2429-2435. DOI: 10.1007/s00417-0173817-4.

4. Aptel F, Denis P, et al. Multicenter clinical trial of high-intensity focused ultrasound treatment in glaucoma patients without previous filtering surgery. Acta Ophthalmol 2016 Aug;94(5):e268-e277. DOI: 10.1111/ aos.12913.

5. Aptel F, Charrel T, et al. Histologic effects of a new device for highintensity focused ultrasound cyclocoagulation. Invest Ophthalmol Vis Sci 2010 Oct;51(10):5092-5098. DOI: 10.1167/iovs.09-5135.

6. Aptel F, Béglé $A$, et al. Short- and long-term effects on the ciliary body and the aqueous outflow pathways of high-intensity focused ultrasound cyclocoagulation. Ultrasound Med Biol 2014 Sep;40(9):2096-2106. DOI: 10.1016/j.ultrasmedbio.2014.04.017. 